Finally, there are hospitals such as the Henderson in Sutton, Surrey, which specialize in the treatment of people with personality disorders who can be either offenders or non-offenders. The principles and philosophy of the therapeutic community obtain. The administration is permissive. Decision making is shared between staff and patients with a view to making the latter assume some responsibility for shaping the world around them. Some measure of success is claimed. The least that can be said for these hospitals is that they are prepared to go on trying.

Freud, or whoever it was, claimed to be able to cure the abnormal "normal" man. Would that he had left us some clue to how to cure the normal "abnormal" man.

\title{
Surgery of Violence
}

\section{Gunshot Wounds of the Limbs}

\author{
R. H. LIVINGSTONE, R. I. WILSON
}

British Medical fournal, 1975, 1, 667-669

\section{Early Management}

The wounds should be dressed with a large pad and bandaged. If there is considerable bleeding a pressure dressing should be applied, and this usually controls most bleeding. If there is a fracture a temporary splint should be used.

The general management at this stage usually includes treatment for shock and blood loss. Intravenous drips must be started and the patient given adequate fluid and blood.

All wounds must be carefully examined. Small superficial wounds should have the skin edges trimmed and a dressing applied. Superficial through-and-through wounds should also have the skin edges excised and the so called "pull-through technique" may be used. This means that forceps are passed through the wound, and a swab soaked in a mild antiseptic such as cetrimide and chlorhexidine (Savlon) is pulled through the wound. This refers to low-velocity injuries with minimal damage to tissues.

\section{DEEP WOUNDS}

These must be thoroughly explored, if necessary by enlarging the wound or in some cases joining the entrance and exit wounds. All dead tissues must be removed, and the dissection may be extensive. The skin edges should be carefully excised. The wound is then left open and packed lightly with tulle. To avoid infection the skin should never be sutured at the initial treatment. Even with the most careful surgery some dead tissue may remain, and there will probably be some haematoma formation. Suturing of wounds at this stage is positively dangerous, and may lead to infection and even to gas gangrene. The wound is normally sutured at five to seven days. This delayed primary suture will produce an excellent result.

\section{WOUNDS OF BONE OR JOINT}

Careful débridement is carried out. Small isolated pieces of bone may be removed, but those with soft tissue attachments must be left. The wound is then treated as already described and a splint applied.

Internal fixation is used only if there has been a concomitant vascular injury requiring vascular repair. It is necessary to fix the fracture to make the limb steady, and allow the vascular repair to heal. If internal fixation is not possible, traction should be used with great caution when a vascular repair has been performed. An injudicious pull on a repaired vessel will narrow its lumen and lead to thrombosis. 
JOINTS

Joint injuries must be carefully explored and all foreign material and loose fragments of bone removed. It may be necessary to wash out the joint with saline to ensure removal of all fragments.

\section{WOUNDS INVOLVING NERVES}

Fortunately most nerve injuries are not permanent. If nerve tissue is lost, there is usually a considerable gap, and this means that primary repair is rarely possible. However, the nerve ends may be marked with a black silk suture to aid identification if delayed repair is carried out.

\section{WOUNDS OF MAJOR VESSELS}

When major blood vessels are damaged it is usual to approach them directly, pressure and packing being applied to control the blood loss until definitive control has been achieved. This can be done in most cases without using Spencer Wells type forceps; DeBakey or bulldog arterial clamps are used together with tapes for intermittent control. Because of the nature of the injuries the incisions are seldom conventional arterial exposures. Deep veins are as important as arteries and they should be dealt with in a similar manner. The urgency to control the haemorrhage means that proper débridement and exploration of the wound is delayed until the haemorrhage is controlled.

The next step with the vessel involves some proximal and distal dissection and possibly the passage of tapes or soft catheters round them. The damaged ends are then trimmed back until healthy tissue is exposed. This may preserve a section of a vessel in continuity as a ribbon, or a gap of varying length may exist.

The content of the vessels distal to the injury should now be considered. In cases which reach the operating theatre within an hour and an obvious back flow of blood occurs from the distal artery $20-40 \mathrm{ml}$ of heparin saline solution is injected distally. In cases arriving any later than this a Fogarty balloon catheter size 3 or 4 is passed before heparin saline is injected. Veins are dealt with similarly. The use of systemic heparin is not advised in such cases.

Fig. 1 illustrates the usual methods available for repairing damaged blood vessels. Simple end-to-end anastomosis is often possible with the limb joints flexed, but this attractive and easy procedure can lead to failure when the joints are extended, and it has been used very rarely. Some failures after this procedure have been retrieved by inserting a graft. The graft should be autogenous vein, since any foreign material is likely to become infected. The source of vein graft can present a problem, particularly as many of these injuries are in the popliteal area and the patient may well be prone. Rarely should the graft be taken from the injured limb, because the last intact venous channel may be sacrificed. Occasionally a proximal length of saphenous vein is taken from the sound limb at the outset, or the arm veins may be used. Flexing the knee allows the distal saphenous vein to be obtained when arm veins are being used for resuscitation of the prone patient.

Fig. 2 shows a proved method of obtaining a graft made from two strips of this rather muscular vein; it functions well in the popliteal and lower femoral arteries. Damaged deep veins should be repaired, and when every vessel has been torn one good deep venous channel should be obtained if possible.

Silk has been the usual suture material, 5/0 atraumatic for arteries and $5 / 0$ or $6 / 0$ for veins. This material is a little more manageable than the smoother synthetic materials in these emergency cases.

Grafted vessels are best covered with neighbouring tissues, and this happens naturally in the majority of cases. Occasionally a catgut suture is necessary to hold a muscle or a fascial layer against a vessel, but otherwise the wound is dealt with in the previously described manner.

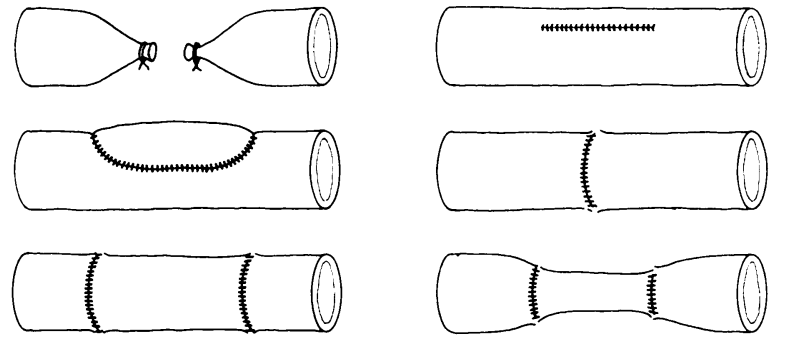

FIG. 1-Top left: ligation illustrated to be avoided. Top right: simple suture seldom possible in arteries but sometimes in veins. Middle left: vein patch. Middle right: end-to-end anastomosis. Bottom left: ideal graft inset difficult to achieve. Bottom right: usual situation with vein graft, and if seriously narrowed can lead to failure.

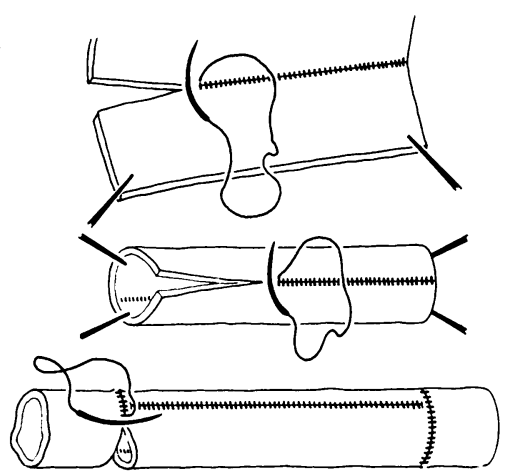

FIG. 2-Method of fabricating a sizable vein graft from two strips of vein. Pin the vein intima side-down on a sterile board and suture longitudinally. Then turn intima inwards and repeat procedure. Finally insert the graft and remember to trim the arteries to fit the graft, because cutting the graft would undo the suture knots and lead to disruption of the graft.

Fasciotomy has not been practised often enough, and severe postoperative oedema has been encountered in muscle compartments, leading to infarction. All cases seen after a delay of several hours should have the anterior tibial compartment decompressed. When muscles above the knee are severely damaged, both the anterior tibial and the posterior tibial compartments should be decompressed by fasciotomy. If one of the tibial compartments is severely damaged by a gunshot wound, the other tibial compartment should be decompressed by fasciotomy.

\section{Later Management}

\section{DELAYED PRIMARY SUTURE}

This technique is imperative in dealing with gunshot wounds. Five to seven days after initial injury the wounds are inspected, and if there is no gross infection they are sutured. Indeed, it may be necessary to make release incisions to allow closure of the original wound. Skin grafting is necessary in many cases and may require several operations.

Severe, extensive injuries, with destruction of the major vessels, nerves, bones, and gross muscle damage, may necessitate primary amputation. Consultation with a colleague before deciding on amputation is advisable.

\section{INFECTION}

With careful surgical techniques infection is not a serious problem. All patients are given antibiotics as a routine. A combination of ampicillin and flucloxacillin is given in full doses until delayed primary suture is performed. In cases with fractures or joint injuries antibiotic treatment may be continued for two to three weeks. 
Gas gangrene has been extremely rare and has occurred only when the wound has been sutured primarily. When it does occur, treatment is by wide excision of the affected tissues combined with gentamicin by intramuscular injection. Hyperbaric oxygen therapy has been available and has been used in these cases.

\section{FRACTURES}

Civil unrest has meant a large increase in the number of fracture cases, and has doubled the average number of fractures of the shaft of the femur. Because of the pressure on beds plaster-ofParis techniques using a number of prosthetic limb principles have been introduced. The quadrilateral ischial bearing long-leg plaster-of-Paris is used for fractures of the femur, and the patellar tendon bearing short-leg walking plaster is used for fractures of the tibia. Patients can be discharged from the ward in a few weeks time, some being in for as short a period as three weeks.

Many of the fractures of the knee joint are carried out as a form of punishment by terrorist organizations. However, the knee-cap itself is rarely injured, but the wound often involves the knee joint or shatters the lower end of the femur and often damages the popliteal vessels or nerves.
NERVE INJURIES

Nerve conduction tests are very helpful in the prognosis of nerve injuries. Most of them are in the nature of neuropraxia and recover in time. But if there is no recovery the nerve may require exploration when all the wounds are healed. If the defect is too large to repair, other procedures such as tendon transposition may be necessary to restore function.

\section{VASCULAR INJURIES}

Secondary haemorrhage has occurred from vascular repairs owing to infection. In a few cases late thrombosis has followed repair of vessels; its association with traction has been mentioned above.

\section{AMPUTATION}

Amputation may be necessary later because of persistent softtissue infection or severe osteomyelitis, particularly if associated with an irrecoverable nerve lesion. Vascular complications such as thrombosis of a graft or delay in attending to a vascular injury until a false aneurysm or arteriovenous fistula forms may necessitate amputation.

\section{Contemporary Themes}

\section{The Emigration of Doctors: A Problem for the Developing and the Developed Countries. Part II*}

\section{B. SENEWIRATNE}

British Medical fournal, 1975, 1, 669-671

\section{Steps Taken to Prevent Doctors from leaving COMPULSORY PERIODS OF SERVICE}

The Parliament of Ceylon in 1961 passed legislation to make public service compulsory for graduates of the University of Ceylon and made specific reference to medical graduates. This law is being strictly enforced for doctors, and a compulsory five-year period of service after qualification is required by law. The effect of this legislation has really never been studied.

The first and most important effect is a loss to the country of graduates with five years' experience rather than those with one year's experience, since as soon as the five-year compulsory period is over doctors who want to go will leave. The loss of a doctor after five years of training means that at about the time that he is able to take full responsibility and could help in the training of others he leaves the country.

Secondly, the erection of a "fence" invariably presents a challenge to the ingenuity of those "fenced in" to find devious means of getting out. The cleverer and more ingenious medical

*Part I appeared in last week's issue.

Faculty of Medicine, University of Sri Lanka, Peradeniya Campus Sri Lanka

B. S ENEWIRATNE, M.D., M.R.C.P., Senior Lecturer in Medicine students leave after the M.B. B.S. examination (while they are still students and before the examination results are released, which could make them doctors).

Finally, a very important effect is that it results in the retention of a group of dissatisfied and frustrated doctors who stay in the country because they are compelled to do so and not because they want to. It cannot be too strongly stressed that an efficient health service cannot be run under these circumstances, and the chaos in the medical services in this country is partly attributable to this.

\section{BONDS}

Those who have been sent abroad for training are being made to sign heavy financial and compulsory service bonds. Surprisingly, this includes university staff who go on sabbatical leave at the end of seven years' service to the country. This shortsighted policy will result in doctors remaining in this country without progressing in their field, since many do not wish to commit themselves to long periods of service (for example, seven to ten years) or the payment of large sums of money.

\section{PERMISSION TO LEAVE THE ISLAND}

Professional people, especially doctors, need the prime minister's approval before they are allowed to leave the country even on a 